\title{
Geometric Proof of Riemann Conjecture
}

\author{
Chuanmiao Chen ${ }^{1,2}$ \\ ${ }^{1}$ School of Mathematics and Statistics, Central South University, Changsha, China \\ ${ }^{2}$ College of Mathematics and Statistics, Hunan Normal University, Changsha, China \\ Email:cmchen@hunnu.edu.cn
}

How to cite this paper: Chen, C.M. (2021) Geometric Proof of Riemann Conjecture. Advances in Pure Mathematics, 11, 334-345. https://doi.org/10.4236/apm.2021.114021

Received: March 6, 2021

Accepted: April 24, 2021

Published: April 27, 2021

Copyright $\odot 2021$ by author(s) and Scientific Research Publishing Inc. This work is licensed under the Creative Commons Attribution International License (CC BY 4.0).

http://creativecommons.org/licenses/by/4.0/

\begin{abstract}
This paper proves Riemann conjecture $(\mathrm{RH})$, i.e., that all the zeros in critical region of Riemann $\xi$-function lie on symmetric line $\sigma=1 / 2$. Its proof is based on two important properties: the symmetry and alternative oscillation for $\xi=u+i v$. Denote $\tau=\beta+i t, \beta=\sigma-1 / 2 \geq 0$. Riemann proved that $\mathrm{u}$ is real and $v \equiv 0$ for $\beta=0$ (the symmetry). We prove that the zeros of $\mathrm{u}$ and $\mathrm{v}$ for $\beta>0$ are alternative, so $u(t, 0)$ is the single peak. A geometric model was proposed. $I_{j}=\left[t_{j}, t_{j+1}\right]$ is called the root-interval of $u(t, \beta)$, if $|u|>0$ is inside $I_{j}$ and $u=0$ is at its two ends. If $|u(t, \beta)|$ has only one peak on each $I_{j}$, which is called the single peak, else called multiple peaks (it will be proved that the multiple peaks do not exist). The important expressions of $\mathrm{u}$ and $\mathrm{v}$ for $\beta>0$ were derived. By $u(t, \beta)=u(t, 0)-\int_{0}^{\beta} u_{t t}(t, r)(\beta-r) \mathrm{d} r$, the peak $u(t, \beta)$ will develop toward its convex direction. Besides, $u_{t}(t, \beta)$ has opposite signs at two ends $t=t_{j}, t_{j+1}$ of $I_{j}, v(t, \beta)=-\int_{0}^{\beta} u_{t}(t, r) \mathrm{d} r$ also does, then there exists some inner point $t^{\prime}$ such that $v\left(t^{\prime}, \beta\right)=0$. Therefore $\{|u|,|v| / \beta\}$ in $I_{j}$ form a peak-valley structure such that $\|\xi\|=|u|+|v| / \beta \geq \mu_{j}(\beta)>0$ has positive lower bound independent of $t \in I_{j}$ (i.e. $\mathrm{RH}$ holds in $I_{j}$ ). As $u(t, \beta)$ does not have the finite condensation point (unless $u$ =const.), any finite $t$ surely falls in some $I_{j}$, then $\|\xi\|>0$ holds for any $\mathrm{t}$ ( $\mathrm{RH}$ is proved). Our previous paper "Local geometric proof of Riemann conjecture" (APM, V.10:8, 2020) has two defects, this paper has amended these defects and given a complete proof of RH.
\end{abstract}

\section{Keywords}

Riemann Conjecture, Geometric Analysis, Symmetry, Alternative Oscillation, Single Peak, Peak-Valley Structure 


\section{Introduction}

D. Hilbert [1] (1900) reported 23 problems in mathematics and stated $\mathrm{RH}$ in the problem 8 as "it still remains to prove the correctness of an exceedingly important statement of Riemann, viz., that the zero points of the function $\zeta(s)$ defined by the series

$$
\zeta(s)=1+\frac{1}{2^{s}}+\frac{1}{3^{s}}+\frac{1}{4^{s}}+\cdots
$$

all have the real part 1/2, except the well-known negative integral real zeros....."

But RH has not been solved in $20^{\text {th }}$ century. Entering new era, S. Smale's report [2] (2000), reviews [3] (2000), [4] (2003) and books [5] [6], all have cited Hilbert's statement. J. Conrey [4] pointed out that "It is my belief, $R H$ is a genuinely arithmetic question that likely will not succumb to methods of analysis". E. Bombieri [3] expected that "For them, we do not have algebraic and geometric models to guide our thinking, and entirely new ideas may be needed to study these intriguing objects". These advices make us realize that the analysis of the infinite series is hopeless and we should pay more attention to the algebraic and geometric analysis. We have also noted a new trend to give up $\zeta$ and turn to $\xi$. P. Sarnak [7] (2004) pointed out that "Riemann showed how to continue zeta analytically in $s$ and he established the Functional Equation:

$$
\Lambda(s):=\pi^{-s / 2} \Gamma\left(\frac{s}{2}\right) \zeta(s)=\Lambda(1-s),
$$

$\Gamma$ being the Gamma function. $\mathrm{RH}$ is the assertion that all the zeros of $\Lambda(s)$ are on the line of symmetry for the functional equation, that is on $\operatorname{Re}(s)=1 / 2$." Where $\Lambda(s)$ has the symmetry and alternative oscillation, which for the first time turns to $\xi$. Besides, J. Haglund [8] (2011) discussed (another form of (1.6))

$$
\Xi(t)=2 \int_{0}^{\infty} \Phi(u) \cos (u t) \mathrm{d} u, \Phi(u)=2 \sum_{n=1}^{\infty}\left(2 a_{n}^{2} \mathrm{e}^{9 u / 2}-3 a_{n} \mathrm{e}^{5 u / 2}\right) \mathrm{e}^{a_{n} \mathrm{e}^{2 u}},
$$

which is the first paper to study the equivalence of $\Xi$ and RH. He computed $\Xi$ and proposed a guess: if any part summation has the monotone zeros, then RH holds. He thought that the study of the $\Xi$-function was the right approach to $\mathrm{RH}$. This is very important.

We have computed the Riemann $\xi$-function and other continuations of Euler $\zeta$-function, and found that only $\xi$ has the symmetry and alternative oscillation, which intuitively implies RH. Whereas others $\zeta$ have no the properties, and proving $\mathrm{RH}$ is hopeless.

We reread the original paper of Riemann (see [5]) and found his thought to study $\xi$-function. We list 4 terms concerning $\mathrm{RH}$ and the important progression as follows.

1) Riemann converted Euler series (1.1) into analytical function (no symmetry)

$$
\zeta(s)=\pi^{s / 2} \Gamma^{-1}\left(\frac{s}{2}\right)\left\{\frac{2}{s(s-1)}+\int_{1}^{\infty}\left(x^{s / 2-1}+x^{-s / 2-1 / 2}\right) \psi(x) \mathrm{d} x\right\} .
$$


where $\psi(x)=\sum_{n=1}^{\infty} \mathrm{e}^{-n^{2} \pi x}$ is Jacobi's function.

2) Introduced an entire function (which is a symmetrization)

$$
\xi(s)=\frac{s(s-1)}{2} \pi^{s / 2} \Gamma\left(\frac{s}{2}\right) \zeta(s) .
$$

In critical domain $\zeta$ and $\xi$ have the same zeros. Taking $s=1 / 2+i t$, using integration by parts twice and Jacobi equality $1 / 2+\psi(1)+4 \psi^{\prime}(1)=0$, Riemann had gotten a real function

$$
\xi(t)=4 \int_{1}^{\infty} \frac{\mathrm{d}\left[x^{3 / 2} \psi^{\prime}(x)\right]}{\mathrm{d} x} x^{-1 / 4} \cos \left(\frac{t}{2} \ln x\right) \mathrm{d} x, \operatorname{Im}(\xi) \equiv 0 .
$$

Remark 1. In present point of view, using translating $\tau=s-1 / 2=\beta+i$, it should directly get Riemann's general formula [5]

$$
\xi(s)=\int_{1}^{\infty}\left(x^{\tau / 2}+x^{-\tau / 2}\right) x^{-3 / 4} f(x) \mathrm{d} x, f(x)=2 x^{2} \psi^{\prime \prime}(x)+3 x \psi^{\prime}(x) .
$$

On critical line $\beta=0, \xi=u+i v$ has the symmetry $v(t, 0)=0$.

3) Riemann said, "The number of roots of $\xi(t)=0$ whose real parts lie between 0 and $T$ is about $=\frac{T}{2 \pi} \ln \frac{T}{2 \pi}-\frac{T}{2 \pi}$ ” (it is proved by Mongoldt, 1905), and pointed out that, "One finds in fact about this many real roots within these bounds and it is very likely that all of the roots are real. One would of course like to have a rigorous proof of this" (i.e. $\mathrm{RH}$ ).

4) He guessed a multiplication formula of $\xi$ (it is proved by Hadamard, 1893)

$$
\xi(t)=\xi(0) \prod_{j=1}^{\infty}\left(1-\frac{t^{2}}{t_{j}^{2}}\right) .
$$

From these we see that Riemann had emphasized $\xi$, rather than $\zeta$.

5) Siegel (1932) found a formula unpublished in Riemann's manuscript(now called R-S formula, which was derived by $\xi$, and is large scale computing formula on critical line up to now), and Riemann had already computed the first several roots (due to the inspiration of R-S formula, we shall propose a new computing formula in next paper).

6) Lagarias [9] (1999) found the positivity $\operatorname{Re}\left(\frac{\xi^{\prime}}{\xi}\right)>0$ for $|\beta|>0$ (a new property undiscussed by Riemann), which is the most essential progression since 1932 , also the first equivalence to $\mathrm{RH}$ for $\xi$. Its proof requires the properties 3) and 4).

7) C. M. Chen [10] [11] (2020) regarded $\xi=u+i v$ as curve family $\{u(t, \beta), v(t, \beta)\}$ with parameter $\beta \geq 0$, and found that in each root-interval $I_{j}$ of $u,\{|u|,|v| / \beta\}$ form a peak-valley structure (a new property undiscussed by Riemann) and $\|\xi\|=|u|+|v| / \beta>0$ (i.e. RH). The framework of geometric analysis used in [11] is correct and should be kept in this paper, but there are two defects in its proof. a) At one end of root-interval $I_{j}$, think $u_{t}\left(t_{j}, \beta\right)>0$ 
to imply $u_{t}\left(t_{j}, r\right)>0, r \in(0, \beta]$ is not strict, which is strictly proved in Lemma 2 of this paper. b) To prove the single peak of $u$, assume that $u$ has multiple peaks, $\|\xi\|>0$ for small $\beta>0$ is proved, but this only is local result and not sufficient for using Lagarias' theorem. This paper directly proves the alternative oscillation of $\{u, v\}$ and derives the single peak of $u$ (Theorem 3). Our main result is

Basic theorem (RC). All the zeros of Riemann $\xi$-function lie on symmetric line.

Therefore this paper has amended the defects and given a complete proof of RC.

\section{Geometric Properties of $\xi$}

\subsection{The Symmetry and Alternative Oscillation of $\xi$}

Denote $\beta=\sigma-1 / 2, \tau=i t+\beta=s-1 / 2$. Consider Riemann $\xi$-integral and its derivatives

$$
\left\{\begin{array}{l}
\xi(s)=\int_{1}^{\infty}\left(x^{\tau / 2}+x^{-\tau / 2}\right) x^{-3 / 4} f(x) \mathrm{d} x=u+i v \\
\xi^{\prime}(s)=\frac{1}{2} \int_{1}^{\infty}\left(x^{\tau / 2}-x^{-\tau / 2}\right) x^{-3 / 4} \ln (x) f(x) \mathrm{d} x=u_{\beta}+i v_{\beta} \\
\xi^{\prime \prime}(s)=\frac{1}{4} \int_{1}^{\infty}\left(x^{\tau / 2}+x^{-\tau / 2}\right) x^{-3 / 4} \ln ^{2}(x) f(x) \mathrm{d} x=u_{\beta \beta}+i v_{\beta \beta}
\end{array}\right.
$$

From decomposing expression

$$
\xi(s)=\int_{1}^{\infty}\left\{\left(x^{\beta / 2}+x^{-\beta / 2}\right) \cos \left(\frac{t}{2} \ln x\right)+i\left(x^{\beta / 2}-x^{-\beta / 2}\right) \sin \left(\frac{t}{2} \ln x\right)\right\} x^{-3 / 4} f(x) \mathrm{d} x
$$

we have

Property 1 (symmetry). The $u(t, \beta)$ is an even function of $\beta$ and $v(t, \beta)$ is an odd function of $\beta$. Taking $\beta=0$, then $v(t, 0) \equiv 0$, i.e.

$$
v=0, u_{\beta}=v_{t}=0, v_{\beta \beta}=-v_{t t}=0, u_{\beta \beta \beta}=-u_{t t \beta}=v_{t t t}=0, \cdots
$$

Property 2 (alternative oscillation). All the zeros of $u$ and $v$ for $\beta>0$ are alternative (proved in theorem 3), see Figure 1. The alternative oscillation intuitively implies RH.

\subsection{A Geometric Model of $\xi$}

Definition 1 (root-interval). For any $\beta \in(0,1 / 2]$, a subinterval $I_{j}=\left[t_{j}, t_{j+1}\right]$ called root-interval of $u$, if the real part $|u(t, \beta)|>0$ inside $I_{j}$, and $u\left(t_{j}, \beta\right)=0, u\left(t_{j+1}, \beta\right)=0$.

Definition 2 (single peak). If $|u|$ has only one peak in each root-interval $I_{j}$, called single peak, unless called multiple peaks(we shall prove no multiple peaks in theorem 3 ).

Using Newton-Leibnitz formula, the symmetry $v(t, 0)=0, u_{\beta}(t, 0)=0$ and Cauchy-Riemann conditions $u_{\beta}=v_{t}, v_{\beta}=-u_{t}$, we have

Lemma 1 (the expression of $v$ ). The image part $v$ of $\xi$ can be expressed by 
$u_{t}$ as

$$
v(t, \beta)=v(t, 0)-\int_{0}^{\beta} u_{t}(t, r) \mathrm{d} r, v(t, 0)=0, \beta \in(0,1 / 2],
$$

Corollary 1. $|v(t, \beta)| / \beta$ is uniformly bounded with respect to $\beta \in(0,1 / 2]$.

Definition 3 (peak-valley structure, PVS). For any $\beta \in(0,1 / 2]$, if $|u|>0$ inside the root-interval $I_{j}=\left[t_{j}, t_{j+1}\right]$, and $v$ has opposite signs at two ends, then there surely exists some inner point $t^{\prime} \in I_{j}$ such that $v\left(t^{\prime}, \beta\right)=0$, and $\{|u|,|v| / \beta\}$ form a peak-valley structure, see Figure 2 .

Lemma 2 (the expression of $u$ ). The real part $u$ of $\xi$ can be expressed by $u_{t t}$ as

$$
\begin{aligned}
& u(t, \beta)-u(t, 0) \\
& =\int_{0}^{\beta} u_{\beta}(t, r) \mathrm{d} r=\left.u_{\beta}(r-\beta)\right|_{0} ^{\beta}-\int_{0}^{\beta} u_{\beta \beta}(t, r)(r-\beta) \mathrm{d} r \\
& =0+d, d=-\int_{0}^{\beta} u_{t t}(t, r)(\beta-r) \mathrm{d} r .
\end{aligned}
$$

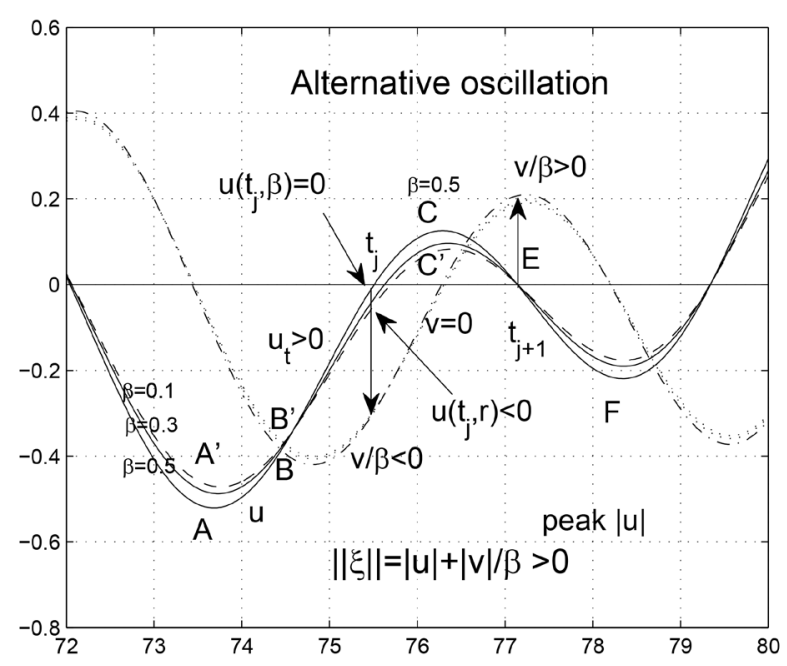

Figure 1. $u_{t}>0$ of curve family $A B C$ and $A^{\prime} B^{\prime} C^{\prime}$.

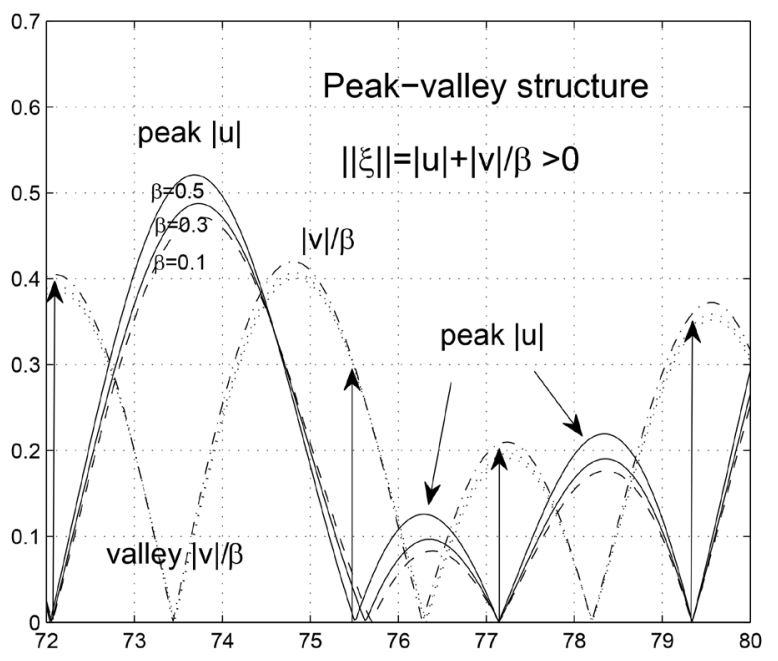

Figure 2. Peak-valley structure. 
Corollary 2. For any $\beta \in(0,1 / 2]$, in the interval of negative peak $u(t, \beta)<0$, with $u_{t t}>0$ (convex downward), then $u(t, \beta)<u(t, 0) \leq 0$. In the interval of positive peak $u(t, \beta)>0$, with $u_{t t}<0$ (convex upward), then $u(t, \beta)>u(t, 0) \geq 0$. So the peak $u(t, \beta)$ will develop toward convex direction (This is the ordering of $u(t, \beta)$ in $\beta$ ).

\subsection{The Slope $u_{t}\left(t_{j}, r\right)$ of Function Family $u(t, r)$}

Consider the curve segments $A B C$ and $C D E$ of $u(t, \beta)$ for $\beta \in(0,1 / 2]$, where $C$ is a positive peak, $A$ and $E$ are negative peaks, see Figure 1 (the varying scale $\xi / M$ is used, $\left.M=8(t / 2)^{23 / 12} \mathrm{e}^{-t \pi / 4}\right)$. Let $I_{j}=\left[t_{j}(\beta), t_{j+1}(\beta)\right]$ be the root interval of $u(t, \beta) \geq 0$, its left end-point $t_{j}=t_{j}(\beta)$ can fall in $A B$ or $B C$, dependent on relative highs of peaks $A$ and $C$. We have

Lemma 2 (the slope $u_{t}$ of curve family). For any $\beta \in(0,1 / 2]$, assume that $u(t, \beta)$ has a positive peak $C$ inside root-interval $I_{j}=\left[t_{j}(\beta), t_{j+1}(\beta)\right]$. If $u(t, \beta)$ is from negative peak to positive one, then curve family $u\left(t, \beta^{\prime}\right)$ on the left line $t=t_{j}(\beta)$ has positive slope

$$
u_{t}\left(t_{j}(\beta), \beta^{\prime}\right)>0 \text {, for any } \beta^{\prime} \in[0, \beta] \text {. }
$$

If $u(t, \beta)$ is from positive peak to negative one, then curve family $u\left(t, \beta^{\prime}\right)$ on the right line $t=t_{j+1}(\beta)$ has negative slope $u_{t}\left(t_{j+1}(\beta), \beta^{\prime}\right)<0$ for any $\beta^{\prime} \in[0, \beta]$. Discussing negative peak is similar.

Proof. For $\beta \in(0,1 / 2]$, the slope $u_{t}>0$ inside $A B C, u_{t t}>0$ inside $A B$, $u_{t t}<0$ inside $B C$ and $u_{t t}=0$ at $B$. Decreasing $\beta^{\prime} \in J=(0, \beta]$, the corresponding curve $A^{\prime} B^{\prime} C^{\prime}$ will continuously vary and the slope $u_{t}>0$ inside $A^{\prime} B^{\prime} C^{\prime}$. To prove $u_{t}\left(t, \beta^{\prime}\right)>0$ for the curve family, we should avoid two peak-points $t_{A^{\prime}}$ and $t_{C^{\prime}}$. Denote the upper bound $p$ of $t_{A^{\prime}}$ and the lower bound $q$ of $t_{C^{\prime}}$ by

$$
p=\max _{\beta^{\prime} \in J} t_{A^{\prime}}\left(\beta^{\prime}\right), \quad q=\min _{\beta^{\prime} \in J} t_{C^{\prime}}\left(\beta^{\prime}\right), \quad \text { for } t \in I^{*}=(p, q) .
$$

Obviously $u_{t}\left(t, \beta^{\prime}\right)>0, \beta^{\prime} \in J$ inside common interval $t \in I^{*}=(p, q)$. We prove the left line $t=t_{j}=t_{j}(\beta) \in I^{*}$, so (2.5) holds.

For this, rewrite the expressions (2.4) for $u(t, \beta)$ and $u\left(t, \beta^{\prime}\right)$ and their difference

$$
\begin{aligned}
& u(t, \beta)-u\left(t, \beta^{\prime}\right) \\
& =-\left\{\int_{\beta^{\prime}}^{\beta} u_{t t}(t, r)(\beta-r) \mathrm{d} r+\int_{0}^{\beta^{\prime}} u_{t t}(t, r)\left(\beta-\beta^{\prime}\right) \mathrm{d} r\right\}, \quad \beta>\beta^{\prime} \geq 0 .
\end{aligned}
$$

Subdivide $A B C$ and $A^{\prime} B^{\prime} C^{\prime}$ into three segments with different convexities. We have

1) If the zero $t_{j} \in\left(\max _{r \in J} t_{B^{\prime}}(r), t_{C}\right)$, in which $u_{t t}<0$, by (2.6), we have the ordering $0=u\left(t_{j}, \beta\right)>u\left(t_{j}, \beta^{\prime}\right)$ and $t_{j}<t_{C^{\prime}}$, see Figure 1 , then $t_{j} \in I^{*}=(p, q)$.

2) If the zero $t_{j} \in\left(t_{A}, \min _{r \in J} t_{B^{\prime}}(r)\right]$, in which $u_{t t}>0$, by (2.6), we have the ordering $0=u\left(t_{j}, \beta\right)<u\left(t_{j}, \beta^{\prime}\right)$ and $t_{j}>t_{A^{\prime}}$, see Figure 1 , then $t_{j} \in I^{*}=(p, q)$. 
3) If the zero $t_{j} \in\left[\min _{r \in J} t_{B^{\prime}}(r), \max _{r \in J} t_{B^{\prime}}(r)\right]$, we can not judge their ordering, but obviously $t_{j} \in I^{*}$.

Above three cases prove (2.5). Similarly discuss the curve $C E F$.

Remark 2. We in [11] intuitively thought that $u_{t}\left(t_{j}, \beta\right)>0$ will imply (2.5), this is not strict. Now (2.5) is strictly proved by three segments of convexity.

\section{Geometric Proof of Riemann Conjecture}

We shall regard $\{u(t, \beta), v(t, \beta)\}$ as a continuously varying process from $\beta=0$ to $\beta=1 / 2$. The zeros $t_{j}^{0}$ of $u(t, 0)$ form an irregular infinite sequence

$$
0<t_{1}^{0}=14.1347<t_{2}^{0}=21.0220<\cdots<t_{j-1}^{0}<t_{j}^{0}<t_{j+1}^{0}<t_{j+2}^{0}<\cdots \rightarrow \infty .
$$

which are single zeros(the double zeros are admitted). For any $\beta \in\left(0, \frac{1}{2}\right]$, the zeros $t_{j}$ of $u(t, \beta)$ form an irregular infinite sequence(dependent on $\beta$ )

$$
0<t_{1}(\beta)<\cdots<t_{j-1}(\beta)<t_{j}(\beta)<t_{j+1}(\beta)<t_{j+2}(\beta)<\cdots \rightarrow \infty .
$$

The initial $I_{j}^{0}=\left[t_{j}^{0}, t_{j+1}^{0}\right]$ continuously becomes to root-interval $I_{j}=\left[t_{j}, t_{j+1}\right]$. We prove

Basic theorem. All zeros of Riemann $\xi$-function lie on critical line.

Its proof consists of three theorems as follows.

Theorem 1. If $u(t, 0)$ is single peak, then peak-valley structure and $R H$ are valid.

Proof. Consider a root-interval $I^{0}=\left[t_{j}^{0}, t_{j+1}^{0}\right]$ of $u(t, 0)$. When increasing $\beta>0$, by corollary 2 , the peak $u(t, \beta)$ will develop toward convex direction. Assuming $u(t, \beta)>0$ inside $I_{j}=\left[t_{j}, t_{j+1}\right]$ for $\beta \in(0,1 / 2]$, we analyze two cases as follows.

At the left end $t_{j}, u\left(t_{j}, \beta\right)=0$ and $u_{t}\left(t_{j}, \beta\right)>0$, by Lemma 2 the slope $u_{t}\left(t_{j}, r\right)>0, r \in(0, \beta] \quad$ (author's remark: in the proof [11] only $u_{t}\left(t_{j}, \beta\right)>0$ is not enough!) we have

$$
\left\{\begin{array}{l}
v\left(t_{j}, \beta\right) / \beta=-\frac{1}{\beta} \int_{0}^{\beta} u_{t}\left(t_{j}, r\right) \mathrm{d} r<0, \\
\lim _{\beta \rightarrow+0} v\left(t_{j}, \beta\right) / \beta=-u_{t}\left(t_{j}, 0\right)<0 .
\end{array}\right.
$$

At the right end $t_{j+1}, u\left(t_{j+1}, \beta\right)=0$ and $u_{t}<0$, similarly,

$$
\left\{\begin{array}{l}
v\left(t_{j+1}, \beta\right) / \beta=-\frac{1}{\beta} \int_{0}^{\beta} u_{t}\left(t_{j+1}, r\right) \mathrm{d} r>0, \\
\lim _{\beta \rightarrow+0} v\left(t_{j+1}, \beta\right) / \beta=-u_{t}\left(t_{j+1}, 0\right)>0 .
\end{array}\right.
$$

They are valid and numerically stable for $\beta \in(0,1 / 2]$.

Because $v(t, \beta)$ has opposite signs at two ends of $I_{j}=\left[t_{j}, t_{j+1}\right]$, there surely exists some inner point $t_{j}^{\prime}=t_{j}^{\prime}(\beta)$ such that $v\left(t^{\prime}, \beta\right)=0$. Then $|v(t, \beta)| / \beta$ is valley, and $\{|u|,|v| / \beta\}$ form a peak-valley structure, see Figure 1. We consider a continuous function of $(t, \beta)$ 


$$
\phi(t, \beta)=|u(t, \beta)|+|v(t, \beta)| / \beta, \beta \in(0,1 / 2], t \in I_{j}=\left[t_{j}, t_{j+1}\right] .
$$

which certainly has a positive lower bound independent of $t \in I_{j}$

$$
\min _{t \in I_{j}} \phi(t, \beta)=\mu_{j}(\beta)>0, \beta \in(0,1 / 2] .
$$

So RH holds in $I_{j}$. This is a refine local geometric analysis.

As each root-interval $I_{j}$ will repeat the peak-valley structure, we get a positive irregular infinite sequence

$$
\mu_{1}(\beta), \mu_{2}(\beta), \cdots, \mu_{j}(\beta), \mu_{j+1}(\beta), \cdots
$$

Because all the zeros $\left\{t_{j}\right\}$ of analytical function $u(t, \beta)$ do not have the finite condensation point (unless $u$ 三const. ), then any finite $t$ surely falls in some $I_{j}$. RH holds for any $t$.

Theorem 2. If two roots of $u(t, 0)$ are very close to each other (including double root), then the peak-valley structure for $\beta>0$ and $R H$ still hold.

Proof. Let $u(t, 0)$ have root-interval $I_{j}^{0}=\left[t_{j}^{0}, t_{j+1}^{0}\right], u(t, 0) \geq 0$ inside $I_{j}$ and $u(t, 0)<0$ outside $I_{j}$, see Figure 3. Assume for $\beta>0, u_{t t}<0$ (convex upward) in larger interval $I \supset I_{j}^{0}$. By (2.4),

$$
u(t, \beta)=u(t, 0)+d, d=-\int_{0}^{\beta} u_{t t}(t, r)(\beta-r) \mathrm{d} r>0, t \in I,
$$

i.e. $u(t, \beta)$ translates $u(t, 0)$ by a positive distance $d$ upward. So $u(t, \beta)$ has a larger root-interval $I_{j}=\left[t_{j}(\beta), t_{j+1}(\beta)\right] \supset I_{j}^{0}$. The $u(t, \beta)$ has a positive peak inside the new interval $I_{j}$. Besides $v(t, \beta)$ has opposite signs at two ends of $I_{j}$ and surely $v(t, \beta)=0$ at some inner point, then $|v| / \beta$ is valley. Therefore $\{|u|,|v| / \beta\}$ form a peak-valley structure and $\|\xi\|>0$ in $I_{j}$.

Remark 3. In large scale computation [12] [13] one found that all the zeros of $u(t, 0)$ are single, no double. Perhaps in the future, double roots can be found. Theorem 2 shows that RH still holds for double roots. Besides one also found

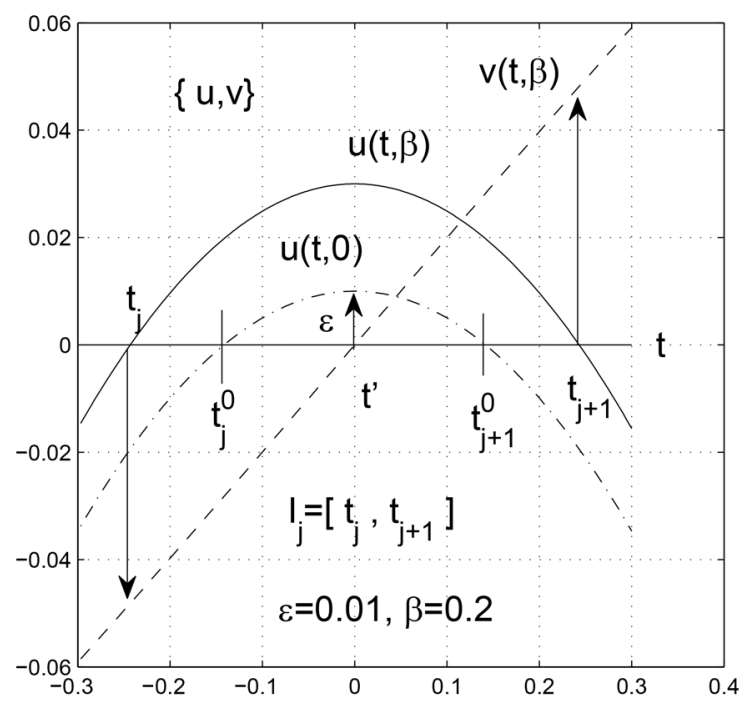

Figure 3. Artificial $u\left(t^{\prime}, 0\right)=\cos (t)-1+\varepsilon$. 
that $u(t, 0)$ is single peak, no multiple one.

Theorem 3. $u(t, 0)$ is single peak.

Proof. We know that analytic function $f(s)=|f(s)|(\cos \psi+i \sin \psi)$ is alternative oscillation with single peak, if $\psi$ monotonously trends to the infinity when $t$ increases. Riemann introduced

$$
\xi(s)=\frac{1}{2} s(s-1) G(s) \zeta(s)=u+i v, G(s)=\pi^{-s / 2} \Gamma(s / 2),
$$

where $G(s)=|G(s)| \mathrm{e}^{i \phi}$, its amplitude

$$
\phi(t)=\frac{t}{2} \ln \frac{t}{2 e \pi}+\left(\beta-\frac{1}{2}\right) \frac{\pi}{4}+O\left(t^{-1}\right),
$$

is super-linearly increasing with $t$, so $G(s)$ is alternative high-frequency oscillation. We recall [5], by amplitude principle, the number of the zeros of $\xi$ in critical rectangle $t \leq T$ is about $N(T)=\frac{T}{2 \pi} \ln \frac{T}{2 e \pi}+O(\ln T)$, its main part is $\phi(T) / \pi$, but $\zeta$ has only $O(\ln T)$. If take an increment $\Delta t=1$ for larger $t$, we have $\Delta \phi=\frac{1}{2}\{\ln t+1-\ln (2 e \pi)\}+O\left(t^{-1}\right)>0$ and $\Delta \psi=O(1)>0$, then the amplitude $\phi+\psi$ monotonously tends to the infinity. As $s(s-1) / 2$ is not oscillation function, it is proved that $G(s) \zeta(s)=|G(s) \zeta(s)| e^{i(\phi+\psi)}$ and then $\xi$ are alternative oscillation with single peak for $\beta>0$. Below prove that its limit value $u(t, 0)$ still is single peak.

By contradiction, assume that $u(t, 0)$ inside root-interval $I_{j}^{0}=\left[t_{j}^{0}, t_{j+1}^{0}\right]$ has three positive peaks $A, B$ and $C$, see Figure 4 , and $u_{t}<0$ at $D$ with $u_{t t}=0$ and $u_{t}>0$ at $E$ with $u_{t t}=0$. So $u_{t}$ at four points $\left\{t_{j}^{0}, t_{D}, t_{E}, t_{j+1}^{0}\right\}$ has the signs $\{+,-,+,-\}$. By (2.3), $v(t, \beta)$ for small $\beta>0$ has the signs $\{-,+,-,+\}$ at these four points, Therefore $v(t, \beta)$ has three different zeros inside $I_{j}$, which

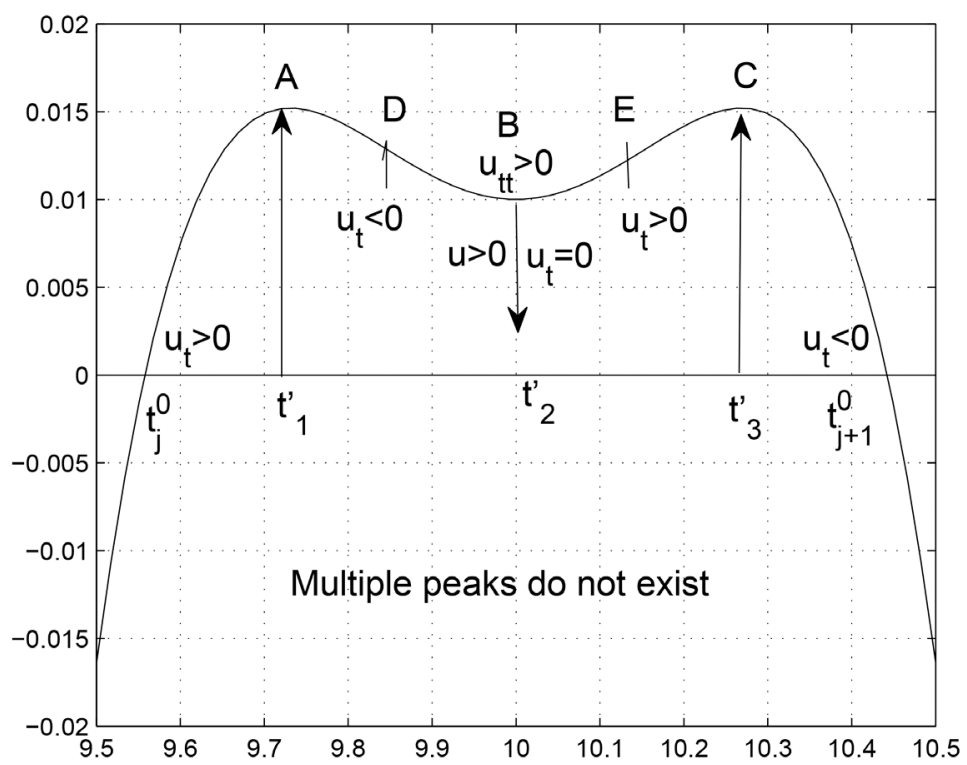

Figure 4. Artificial curve (do not exist). 
goes against the alternative oscillation and then $u(t, 0)$ is single peak (Author's remark: in multiple peak case, [11] proved $\|\xi\|>0$ for small $\beta>0$, but this is only a local result, which is not sufficient for using Lagarias' theorem. This paper directly proves theorem 3 )

Finally summarizing three theorems above, our basic theorem (RH) is proved.

It is interesting that the proof of $\mathrm{RH}$ looks like to solve the Cauchy problem of Cauchy-Riemann system $u_{\beta}=v_{t}, v_{\beta}=-u_{t}$ with analytic initial values $u(t, 0), v(t, 0)=0$ on line $\beta=0$. Follow Riemann, by $\tau=\beta+i t=i z, z=t-i \beta$, its solution is $\xi(\tau)=u(z, 0)$.

\section{Three Corollaries of Lagarias Theorem}

Assume that RH holds, Lagarias (1999) proved the following wonderful result:

Lagarias theorem. $R H$ is equivalent to the positivity $R e\left(\frac{\xi^{\prime}}{\xi}\right)>0$ for any $\beta>0$.

Denoting $\tau=\beta+i t$ and $\xi(\tau)=u+i v$, the positivity can be expressed in the form

$$
\operatorname{Re}\left(\frac{\xi^{\prime}}{\xi}\right)=\operatorname{Re}\left(\frac{\xi^{\prime} \bar{\xi}}{|\xi|^{2}}\right)=\frac{\psi(t)}{|\xi|^{2}}>0, \psi(t)=u u_{\beta}+v v_{\beta}>0, u_{\beta}=v_{t}, v_{\beta}=-u_{t} .
$$

This result is sharp (can not be improved), which has three important corollaries.

Corollary 3. The peak-valley structure and $R H$ are equivalent.

Proof. Above RH is proved by peak-valley structure. Now assume that RH holds, then the positivity also is valid, we shall prove peak-valley structure. Let $I_{j}=\left[t_{j}, t_{j+1}\right]$ be root-interval of $u(t, \beta), u>0, u_{t t}<0$ inside $I_{j}$, and $u_{t}\left(t_{j}, \beta\right)>0, u_{t}\left(t_{j+1}, \beta\right)<0$.

At the left end $t_{j}, u=0, u_{t}>0$, then $\psi=u v_{t}-v u_{t}=-v u_{t}>0$ leads to $v<0$.

At the right end $t_{j+1}, u=0, u_{t}<0$, then $\psi=u v_{t}-v u_{t}=-v u_{t}>0$ leads to $v>0$.

Then $|v|$ is valley in $I_{j}$ and $\{|u|,|v|\}$ form peak-valley structure.

Therefore the peak-valley structure is the exact geometric description to $\mathrm{RH}$.

Corollary 4. If RH holds, then $u(t, 0)$ is single peak.

Proof. If $u(t, 0)$ has three peaks, see Figure 4 , and $u>0, u_{t}=0, u_{t t}>0$ and $v \equiv 0$ at extremes point $t_{2}^{\prime}$. For small $\beta>0$, the $u_{t}\left(t^{\prime}, \beta\right)=0$ at some point $t^{\prime}$ near $t_{2}^{\prime}$, and in its neighbor, $u>0, u_{t t}>0$ and

$$
v_{t}\left(t^{\prime}, \beta\right)=0-\int_{0}^{\beta} u_{t t}\left(t^{\prime}, r\right) \mathrm{d} r<0 .
$$

At this point $t^{\prime}, \psi(t)=u v_{t}-v u_{t}=u v_{t}<0$ is contradiction to the positivity.

So we see that the correctness of our geometric proof of RH can be derived by Lagarias' theorem. 
Corollary 5 (Monotone). If $R H$ holds, then $|\xi(t, \beta)|>\left|\xi\left(t, \beta_{0}\right)\right| \geq 0$ for $\beta>\beta_{0} \geq 0$.

Proof. Using (4.1), the positive integral

$$
|\xi(t, \beta)|^{2}-\left|\xi\left(t, \beta_{0}\right)\right|^{2}=2 \int_{\beta_{0}}^{\beta}\left(u u_{\beta}+v v_{\beta}\right) \mathrm{d} \beta>0 \text {, for } \beta>\beta_{0} \geq 0,
$$

means the monotone. So $|\xi(t, \beta)|>0$ for $\beta>0$ is a concise statement of RH.

Remark 4. We recall that Aristotle, an ancient Greek philosopher and mathematician, thought: "order and symmetry are important elements of beauty". Therefore we can say, the symmetry and monotone of $\xi$ are mathematical beauty of Riemann conjecture.

\section{Acknowledgements}

The author expresses sincere gratitude to the reviewer for his careful remark, valuable and constructive comments. Besides, I should thank Prof. Zhengtin Hou and Prof. Xinwen Jiang for their precious opinion in the discussion.

\section{Conflicts of Interest}

The author declares no conflicts of interest regarding the publication of this paper.

\section{References}

[1] Browder, F. (1976) Mathematical Developments Arising from Hilbert Problems. American Math Society, Providence, RI. https://doi.org/10.1090/pspum/028.1

[2] Smale, S. (1998) Mathematical Problems for Next Century. The Mathematical Intelligencer, 20, 7-15 (also see, Mathematics: Frontiers and Perspectives. American Mathematical Society, Providence, 2000, 271-294). https://doi.org/10.1142/9789812792815_0045

[3] Bombieri, E. (2000) Problems of the Millennium: The Riemann Hypothesis. http://www.claymath.org

[4] Conrey, J. (2003) The Riemann Hypothesis. Notices of the American Mathematical Society, 50, 341-353.

[5] Edwards, H.M. (2001) Riemann’s Zeta Function. Dover Publication, Mineola.

[6] Borwein, P., Choi, S., Rooney, B. and Weirathmuller, A. (2008) The Riemann Hypothesis. Springer, New York.

[7] Sarnak, P. (2004) Problems of the Millennium: The Riemann Hypothesis. http://www.claymath.org

[8] Haglund, J. (2011) Some Conjectures on the Zeros of Approximates to the Riemann $\Xi$-Function and Incomplete Gamma Functions. Central European Journal of Mathematics, 9, 302-318. https://doi.org/10.2478/s11533-010-0095-3

[9] Lagarias, J. (1999) On a Positivity Property of the Riemann $\xi$-Function. Acta Arithmetica, 89, 213-234. https://doi.org/10.4064/aa-89-3-217-234

[10] Chen, C.M. (2020) The Symmetry of Riemann $\xi$-Function. Advances in Pure Mathematics, 10, 464-470. https://doi.org/10.4236/apm.2020.108028 
[11] Chen, C.M. (2020) Local Geometric Proof of Riemann Conjecture. Advances in Pure Mathematics, 10, 589-610. https://doi.org/10.4236/apm.2020.1010036

[12] Lune, J. and Riele, H. (1983) On the Zeros of the Riemann Zeta Function in the Critical Strip. Part 3. Mathematics of Computation, 41, 759-767.

https://doi.org/10.1090/S0025-5718-1983-0717719-3

[13] Lune, J., Riele, H. and Winter, D. (1986) On the Zeros of the Riemann Zeta Function in the Critical Strip. Part 4. Mathematics of Computation, 46, 667-681.

https://doi.org/10.1090/S0025-5718-1986-0829637-3 